\title{
Fish-eye Optics for the Scanning Electron Microscope
}

\author{
T. Agemura ${ }^{* * *}$ and D. C. Joy ${ }^{* * * *}$ \\ * EM Facility, University of Tennessee, Knoxville, TN 37996 \\ ** Hitachi High-Technologies Corporation, Hitachinaka, 312-8504 Japan \\ *** Oak Ridge National Laboratory, Oak Ridge, TN 37981
}

'Fish-eye' lenses are used in conventional light optics to produce images containing a very wide angle of view. When mounted with the lens axis vertical then an image spanning +/- 60 degrees about the axis, and 360 degrees azimuthally can be obtained although at the cost of some image distortion. There is no direct electron-optical equivalent to the fish-eye configuration because of the need to use all such lenses with a small numerical aperture to minimize aberrations. However, it is well known in the scanning electron microscope (SEM) that if a specimen is allowed to charge up to such a high energy that it acts as an electron mirror then the 'reflection' of the scanning beam from the surface produces a wide angle image of the inside of the specimen chamber.

We report here on a new optical arrangement which provides SEM images analogous to those from a fish-eye lens. The arrangement is shown schematically in FIG. 1 and the novel element in the system is a permanent magnet 'lens'. The item used here is a Neodymium Iron Boron ( $\mathrm{NdFeB})$ disc 0.25 inches in diameter and 0.1 inches thick.[1] The magnet is made by a powder forming process and so has a granular surface which is rough and chemically reactive. To protect the surface, and to minimize irregularities in the magnetic field the surface is covered with electrochemically deposited nickel. Other shapes are possible to fabricate but machining is difficult because the $\mathrm{NdFeB}$ is extremely hard and brittle. Since even small $\mathrm{NdFeB}$ magnets have exceptionally high levels of magnetization compared to the fields obtainable from a conventional electro-magnet, they are very suitable for use as an electron lenses.[2] They must, however, be treated with care as even a disc as small as the one used here can lift several kilograms and once attracted to a metal surface it is all but impossible to remove. The magnet used here was formed during manufacture to provide a dipole along the cylindrical axis of the disc, and is positioned in the column with the magnet axis collinear with the optic axis of the SEM and with the magnetic North pole facing upwards

FIG. 1 also illustrates a simulation result of electron trajectories.[3] The dipole permanent magnet which has a magnetization of 1 Tesla was mounted on the specimen stage set at $43 \mathrm{~mm}$ in working distance and at $25 \mathrm{~mm}$ in $\mathrm{X}$ direction. Green lines are representative of magnetic fields. Electron trajectories can be widely changed by moving the stage. FIG. 2 shows a secondary electron image inside the specimen chamber of Hitachi S-4300SE SEM taken in the 'Fish-eye' lens mode by using the NdFeB disc. Three detectors (EBS, EDX and BSE), the objective lens and the chamber wall are clearly seen in the image. Primary electrons can be easily focused on dhamber wall features.

\section{References}

[1] The magnet was obtained from ForceField Inc, 2606 West Vine Dr., Fort Collins, CO 80521.

[2] A. V. Crewe and D. Kielpinski, Optik 103 (No. 4) (1996) 167.

[3] The Simulation program was developed by Mr. Y. Ose who is a senior engineer of Hitachi High-Technologies Corporation. 


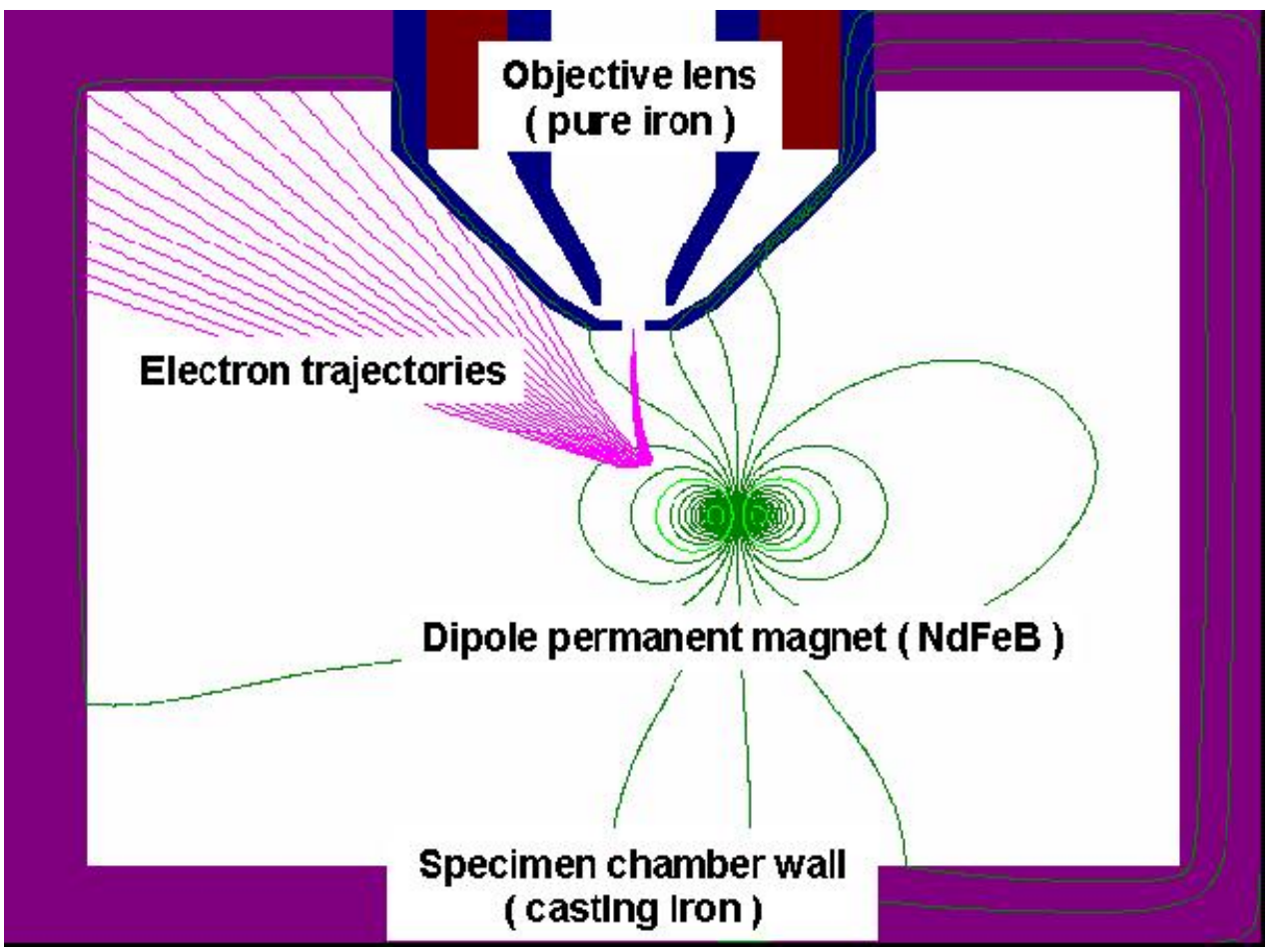

FIG. 1. A New optical arrangement analogous to 'Fish-eye' lens arrangement for the SEM and a simulation result of electron trajectories.

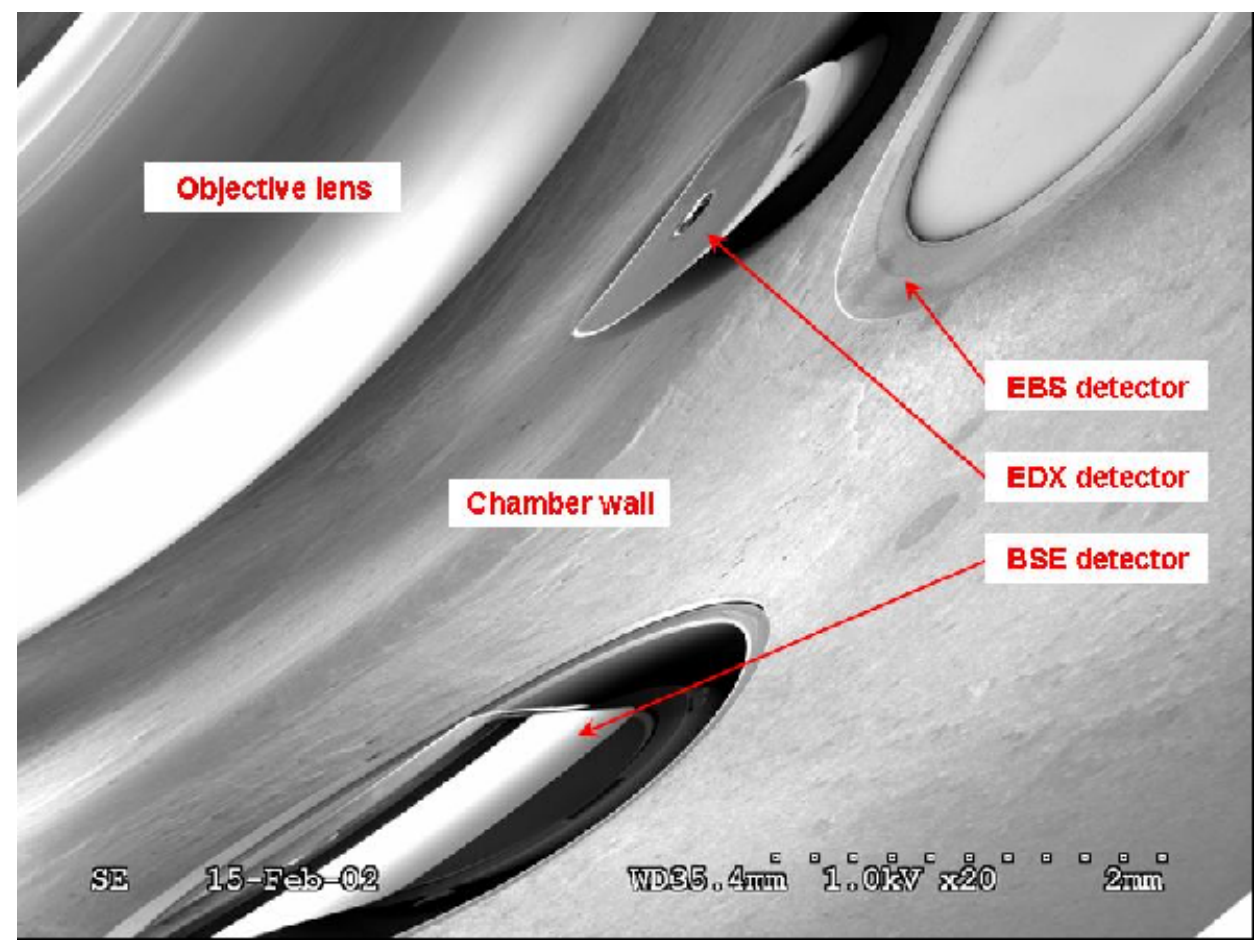

FIG. 2. A secondary electron image inside the specimen chamber of Hitachi S-4300SE SEM taken in the 'Fish-eye' lens mode by using the NdFeB disc. 